\title{
Targeted Ion Milling of Ex Situ Lift-Out FIB Specimens
}

\author{
C.S. Bonifacio ${ }^{1}$, M.J. Campin ${ }^{1}$, P. Nowakowski ${ }^{1}$, M. Boccabella ${ }^{1}$, L.A. Giannuzzi ${ }^{2}$ and P.E. Fischione ${ }^{1}$ \\ 1. E.A. Fischione Instruments Inc., Export, PA 15632 USA \\ 2. EXpressLO LLC, Lehigh Acres, FL 33971 USA
}

Transmission electron microscopy (TEM) specimens are typically prepared using a focused ion beam (FIB) because it provides accuracy and site-specificity for thinning and extraction [1]. Nowadays, FIB ex situ lift-out (EXLO) TEM preparation is employed due to its higher throughput and flexibility when compared to traditional in situ FIB preparation [2]. A variety of specimen thicknesses can be prepared with no redeposition artifacts in a fast and reproducible manner. However, $\mathrm{Ga}^{+}$ion milling may cause artifacts such as surface damage and ion-implanted layers, which subsequently limits analytical and high-resolution electron microscopy. Low energy $(<1 \mathrm{keV}) \mathrm{Ar}^{+}$ion milling has been shown to improve TEM specimen quality [3, 4]. Here, we present targeted ion milling using a Fischione Instruments PicoMill TEM specimen preparation system to prepare electron transparent and Ga-free EXLO FIB TEM specimens.

Si specimens with thickness between 200 - 300 nm were prepared using an FEI 200 FIB workstation operated at $30 \mathrm{keV}$. These Si specimens were then manipulated to EXpressLO grids (Figure 1a) outside the FIB and in ambient conditions [2]. Specimens were secured to the grid by Van der Waals forces (i.e., ion beam deposition or glue was not needed) [2]. Ion milling was carried out on each specimen side using the PicoMill system with an ion beam spot size of $600 \mathrm{~nm}$ and decreasing ion energies (Figure 1b). TEM images and energy-filtered TEM (EFTEM) thickness maps were acquired using an FEI Tecnai F30 operated at $300 \mathrm{kV}$. Energy dispersive X-ray spectroscopy (EDS) was performed using an Oxford Instruments X-Max $150 \mathrm{~mm}^{2}$ EDS detector and LayerProbe Aztec Energy software for chemical analyses and thickness evaluation before and after milling.

Thickness reduction by contrast change with decreasing ion beam energy was observed in situ using both the secondary electron detector and STEM detector in the PicoMill system (Figure 1b). Precise beam positioning prevented the redeposition of sputtered material in the 3 to $4 \mu \mathrm{m}$ opening of the grid. After milling, no visible surface damage, i.e., no halo on the Fast Fourier Transform (FFT) of the highresolution TEM (HRTEM) image (Figure 2b) was observed. EDS comparison results confirm elimination of Ga damage after PicoMilling (Figure 2c). The $30 \mathrm{keV} \mathrm{Ga}^{+}$ions produce $22 \mathrm{~nm}$ of $\mathrm{Si}$ sidewall damage [5] while the thickness of detectable Ga implantation via EDS is confined to $<10 \mathrm{~nm}$ [6]. Thickness analysis results show that the $275 \mathrm{~nm}$ thick specimen with a $2 \mathrm{~nm}$ detectable Ga layer per side was reduced to $12 \mathrm{~nm}$ thickness with no detectable Ga after ion milling, effectively removing the Ga implanted and damage region. Quantitative analysis of redeposition from the TEM grid and/or capping layer during targeted milling is underway [7].

\section{References:}

[1] LA Giannuzzi and FA Stevie, Micron 30 (1999), p. 197.

[2] LA Giannuzzi et al, Microscopy and Microanalysis 21 (2015), p.1034.

[3] KA Unocic, MJ Mills, and GS Daehn, Journal of Microscopy 240 (2010), p. 227.

[4] RR Cerchiara et al, Microscopy Today 19 (2011), p. 16. 
[5] LA Giannuzzi, R Geurts, and J Ringnalda, Microscopy and Microanalysis 11 (2005) p.828.

[6] T. Ishitani et al, Journal of Vacuum Science and Technology B 16 (1998) p.1907.

[7] LA Giannuzzi would like to thank TSS Microscopy for granting access to the FIB 200 used in this work.

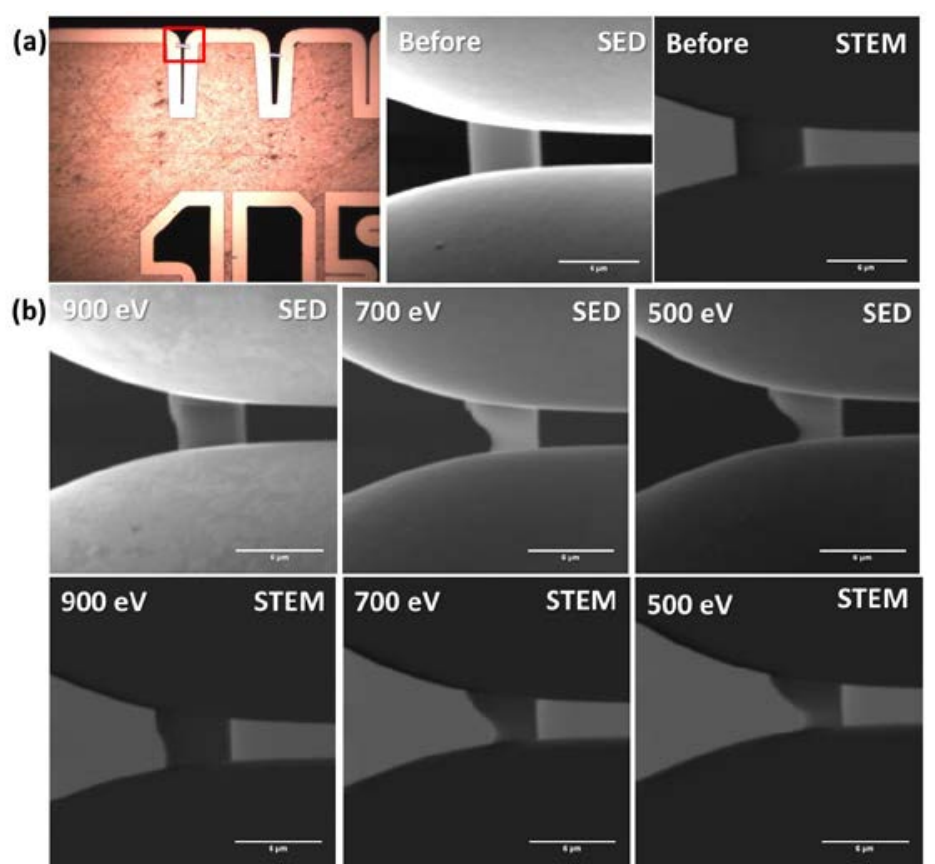

Figure 1. (a) Ex situ lift-out Si specimen on EXpressLO grid (left) before ion milling (center and right). (b) Imaging in the PicoMill system using SED and STEM showing the thickness reduction of the Si specimen during $\mathrm{Ar}^{+}$ion milling.
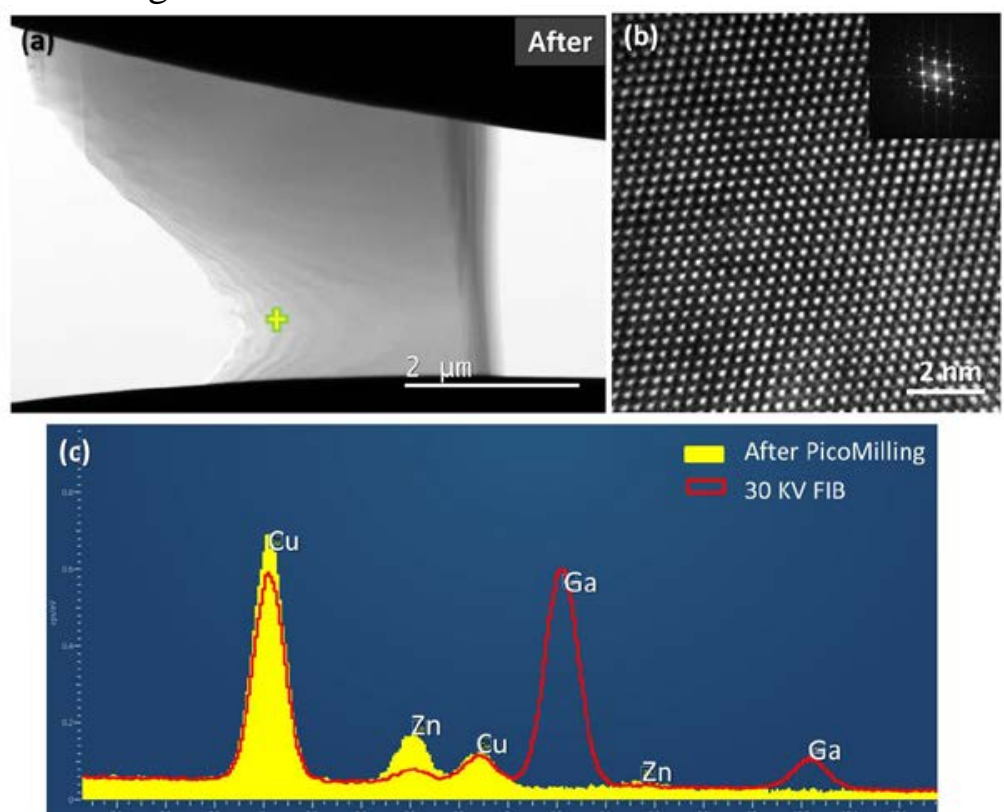

Figure 2. (a) Low magnification TEM image and (b) HRTEM image with corresponding FFT show an electron-transparent specimen that was free from surface damage after ion milling. EDS data (c) were acquired at $15 \mathrm{kV}$ and 240 seconds confirmed the complete removal of Ga from the specimen. Zn and $\mathrm{Cu}$ are from the specimen mount and TEM grid, respectively. Marked area in (a) shows the area for EDS acquisition after the milling process. 